\title{
X Workshop sobre Aspectos da Interação Humano-Computador para a Web Social
}

\author{
Kamila Rios H. Rodrigues \\ Instituto de Ciências Matemáticas e de Computação \\ ICMC/USP \\ São Carlos - SP - Brasil \\ kamila.rios@icmc.usp.br
}

\author{
Rodrigo Pereira dos Santos \\ Departamento de Informática Aplicada \\ UNIRIO \\ Rio de Janeiro - RJ - Brasil \\ rps@uniriotec.br
}

\section{RESUMO}

Pela Web Social, é possível a conexão entre pessoas, organizações e conceitos. Ela desempenha um papel central ao possibilitar que diversas pessoas interajam de diferentes formas e em escalas inéditas por meio de diferentes aplicações, construídas com diversas plataformas e embutidas em variados dispositivos. A décima edição do WAIHCWS pretende promover a continuidade das discussões sobre as oportunidades e os desafios que a Web Social traz para a comunidade de pesquisa em Interação HumanoComputador (IHC) no Brasil e no mundo, colocando em evidência, especialmente neste ano, o tema "10 edições do WAIHCWS: retrospectiva, tendências e novos desafios". O tema representa uma oportunidade para analisar o caminho percorrido por essa comunidade até aqui, bem como os avanços alcançados e os desafios que ainda precisam ser superados tendo em vista as novas tecnologias e formas de interação.

\section{PALAVRAS-CHAVE}

Web Social, Interação Humano-Computador, 10 edições.

\section{TEMA ESPECÍFICO}

A última década tem sido marcada pela presença de novas tecnologias que estão cada vez mais acessíveis e enraizadas no cotidiano das pessoas. Tais tecnologias trazem consigo mudanças na forma de interagir dos indivíduos e nas suas relações sociais. A comunidade científica tem explorado aspectos da interação humana, assim como o design de interfaces e o desenvolvimento de sistemas, apontando desafios sociotécnicos na rede social [1]. A Web, por sua vez, também passa por transformações constantes e vem se adaptando às novas formas de interação promovidas pelo uso dos novos dispositivos.

Alinhada a esse novo contexto, a comunidade de IHC tem repensado suas práticas buscando assim, entender melhor essas novas formas de interação e como empregar suas técnicas no processo de concepção e avaliação de soluções computacionais,

Permission to reproduce or distribute, in whole or in part, material extracted from this work, verbatim, adapted or remixed, as well as the creation or production from the content of such work, is granted without fee for non-commercial use, provided that the original work is properly credited.

IHC 2019 - WORKSHOPS, outubro 21-25, 2019, Vitória, Brasil. In Anais Estendidos do XVIII Simpósio Brasileiro sobre Fatores Humanos em Sistemas Computacionais. Porto Alegre: SBC.

(C) 2019 by the author(s), in accordance with the terms of the Creative Commons Attribution-NonCommercial 4.0 International Public License (CC BY-NC 4.0). engajando cada vez mais os usuários alvo [2, 3] nesse processo.

O XVIII Simpósio Brasileiro sobre Fatores Humanos em Sistemas Computacionais (IHC 2019) põe em pauta o tema: Pontes para Interação. $\mathrm{O}$ evento tem o objetivo de "enfatizar a necessidade de transpor barreiras, cruzar fronteiras, agregar disciplinas, ligar passado e futuro, físico e digital, arte e ciência, para melhorar a qualidade da interação entre pessoas e tecnologias". Entre os tópicos de interesse do simpósio estão: IHC na sociedade (e.g. acessibilidade, gêneros, minorias e aspectos sociais); Áreas específicas de aplicação da IHC (e.g. cidades/ambientes inteligentes e redes/sistemas sociais); Técnicas e estilos de interação (e.g. Interfaces inteligentes, adaptáveis e adaptativas, interação sensível ao contexto e novos Paradigmas de Interação); e Interação entre áreas (e.g. Computação Ubíqua, Educação, Mídias Sociais e Sustentabilidade). Outro tópico de interesse que o simpósio inclui são os grandes desafios de pesquisa em IHC no Brasil - GranDIHC-BR.

Nesse sentido, o tema proposto para o WAIHCWS 2019 busca contribuir com a discussão dos tópicos supracitados, evidenciando os 10 anos do workshop e as conquistas, tendências, bem como os desafios da Web Social frente às novas formas de interação e novas tecnologias. É ainda uma oportunidade de discutir os avanços na área e fazer uma retrospectiva de como a comunidade de IHC tem conduzido pesquisas neste tema.

\section{TÓPICOS DE INTERESSE}

Com foco na Web Social e considerando suas interseções com o tema central desta edição do workshop (10 edições do WAIHCWS: retrospectiva, tendências e novos desafios), os tópicos de interesse incluem, mas não estão restritos a:

- Métodos de design e de avaliação que considerem questões sociais e de valores humanos;

- Compartilhamento de contexto em software social e soluções para promover a interação social;

- Mobilidade, ubiquidade e reflexo na interação humana;

- Adaptação de software social para dispositivos móveis;

- Teorias e mecanismos de incentivo à participação e colaboração dos usuários;

- Fatores, características e medidas de sucesso em software social e em plataformas de ecossistemas de software;

- Aspectos culturais, de apropriação, emocionais, afetivos e o estímulo ao comportamento sustentável; 
- Estratégias e mecanismos de visualização;

- Comunicação, colaboração e compartilhamento;

- Auditabilidade, confiança e reputação em redes sociais;

- Ética no design (projeto, desenvolvimento, avaliação) e uso de soluções computacionais;

- Identidade, privacidade e segurança;

- Usabilidade e acessibilidade;

- Memória, legado e propriedade em softwares sociais;

- Aproveitamento do conhecimento social;

- Modelos de desenvolvimento de software baseados em comunidade, como open source, crowdsourcing e parcerias público-privadas em ecossistemas de software;

- Uso de redes sociais e mídias sociais por equipes de software;

- Gestão do conhecimento e aprendizagem organizacional aliadas aos aspectos técnicos, humanos, sociais e organizacionais no desenvolvimento de software.

- Sustentabilidade na Web e na Computação;

- Interação e integração entre as diferentes áreas do conhecimento.

\section{HISTÓRICO}

A primeira edição do WAIHCWS foi realizada no IHC'08 e teve como objetivo iniciar a discussão em torno do tema pela comunidade brasileira. $\mathrm{O}$ foco da $1^{\mathrm{a}}$ edição esteve na análise dos sistemas colaborativos e de aspectos relacionados ao comportamento dos usuários, em especial no estudo e projeto de aplicações; no gerenciamento de conhecimento; em recomendação; interação; e em aspectos de identidade dos usuários. O WAIHCWS'08 contou com a participação de mais de 50 pessoas. De um total de 13 trabalhos submetidos, foram selecionados 7 para apresentação e publicação ${ }^{1}$.

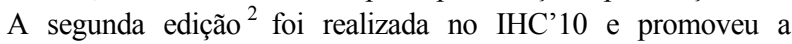
continuidade das discussões sobre as oportunidades e os desafios de pesquisa no contexto da Web Social, colocando em foco os possíveis efeitos em conceitos de sociabilidade e valores humanos. O WAIHCWS'10 contou com a participação de aproximadamente 35 pessoas. Foram recebidos 14 trabalhos (12 artigos completos e 2 artigos resumidos), sendo 12 do Brasil, um da França e um da Índia. Seis trabalhos foram aceitos para publicação. Nesta edição, destacaram-se os seguintes pontos: i) a formação de um comitê de programa internacional; ii) os artigos foram publicados nos anais estendidos do IHC'10 e os três artigos com melhor avaliação pelo comitê de programa foram convidados para submissão em versão estendida à Revista de Informática Teórica e Aplicada.

A terceira edição ${ }^{3}$ do WAIHCWS foi realizada no IHC'11 e teve como proposta oferecer um espaço de discussão sobre como os fatores organizacionais e culturais de uma sociedade em transformação influem na Web Social. Foram submetidos 12 artigos, dos quais 6 foram avaliados e aceitos por um comitê de programa internacional. Estes trabalhos foram publicados online através do site CEUR Workshop Proceedings ${ }^{4}$. O evento contou com 13 participantes e sua agenda incluiu a realização de dinâmicas para fomentar e enriquecer a discussão entre os participantes.

\footnotetext{
http://www.din.uem.br/gsii/i-waihcws-ihc08/

http://www.ic.unicamp.br/ waihcws/

3 http://www2.dc.ufscar.br/waihcws/

4 http://ceur-ws.org/Vol-797/
}

A quarta edição do WAIHCWS foi realizada no IHC'12 e teve como tema a mobilidade e ubiquidade na Web Social, seus desafios e oportunidades. Foram submetidos 15 artigos, dos quais 5 foram aceitos por um comitê de programa internacional. Todos os trabalhos foram publicados online através do site CEUR Workshop Proceedings ${ }^{5}$. O evento contou com 17 participantes e recebeu como palestrante internacional.

A quinta edição do WAIHCWS foi realizada no IHC'13 e teve como tema a inteligência coletiva e a deliberação participativa na Web Social. Foram submetidos 16 artigos, todos brasileiros, dos quais 7 foram aceitos por um comitê de programa internacional. Foi realizada uma dinâmica para projeto de soluções para a Copa do Mundo com base nos elementos da Web Social e com uso de personas. Todos os trabalhos foram publicados online através do site CEUR Workshop Proceedings ${ }^{6}$. O evento contou com 23 participantes de diversos estados brasileiros.

A sexta edição do WAIHCWS foi realizada no IHC'15 e teve como tema o entendimento e a avaliação das informações na Web Social. Dentre os artigos submetidos, 5 foram selecionados por um comitê de programa internacional para apresentação no evento. Além disso, o autor de um trabalho do evento principal foi convidado a apresentá-lo no workshop e participar das discussões com os demais participantes do evento, devido à afinidade do assunto abordado por ele com o tema do evento. Todos os trabalhos foram incluídos nos Anais Estendidos do IHC 2016 e foram publicados online na BDBComp ${ }^{7}$. O evento contou com 19 participantes de diversos estados brasileiros. Foi realizada uma dinâmica na qual os participantes auditaram a credibilidade de informações na Web Social.

A sétima edição do WAIHCWS aconteceu no IHC'16 em São Paulo e foi realizada em dois períodos. Na primeira parte foram apresentados 11 artigos e então discutidos ao final da seção com uma dinâmica. A segunda parte foi uma prática imersiva no Cemitério da Consolação, onde o grupo fez uma visita guiada, acessando os QRCodes dos túmulos e fazendo uso da aplicação que dá acesso a dados dos falecidos, o MemoriAll ${ }^{8}$, via dispositivos móveis. A dinâmica permitiu articular a temática do workshop com discussões teóricas e atividades práticas, trazendo elementos reais relacionados à ética, privacidade, legado digital, e aos valores humanos, tanto de uma perspectiva universal, quanto culturalmente situada. O workshop contou com a participação de cerca de 30 pessoas, das quais 21 participaram da parte externa. Todos os trabalhos foram publicados online no CEUR Workshop Proceedings ${ }^{9}$.

A oitava edição do WAIHCWS foi realizada em Joinville no IHC'17 em um período dividido em duas sessões. A primeira sessão foi composta por uma palestra sobre "Ecossistemas de Software \& Inovação na Web Social”. A palestra foi motivada pelo tema desta edição ter sido "relações técnicas, humanas e organizacionais em ecossistemas de software" na perspectiva do desenvolvimento tecnológico de qualidade. Ainda nesta sessão, foram apresentados

\footnotetext{
http://ceur-ws.org/Vol-980/

6 http://ceur-ws.org/Vol-1051/

${ }^{7}$ http://www.lbd.dcc.ufmg.br/bdbcomp/servlet/Evento?id=859

8 http://www.memoriall.com.br/

9 http://ceur-ws.org/Vol-1714/
} 
X Workshop sobre Aspectos da Interação Humano-Computador para a Web Social

alguns trabalhos selecionados. Na segunda sessão, outros trabalhos foram apresentados além de ter sido realizada uma discussão para criar o mapa de pesquisa em Web Social no Brasil. Foram submetidos 14 trabalhos, sendo que 8 foram aceitos. O workshop contou com a participação de cerca de 20 pessoas. Todos os trabalhos foram publicados online no CEUR Workshop Proceedings ${ }^{10}$.

A nona edição do WAIHCWS foi realizada no IHC'18 em Belém/PA com o tema relacionado à sustentabilidade e fomentou a discussão sobre a Web e as relações sociais, econômicas e ambientais. $\mathrm{O}$ tema foi inspirado em um dos Grandes Desafios de Pesquisa em IHC no Brasil: Futuro, Cidades Inteligentes e Sustentabilidade. 20 trabalhos foram submetidos e 12 foram aceitos. O workshop foi realizado em dois períodos: no primeiro foram conduzidas duas sessões técnicas, cada uma com a apresentação de quatro trabalhos e, o no segundo período, ocorreu uma sessão técnica, também com quatro trabalhos, e um painel que promoveu a discussão sobre o tema proposto e contou com a participação de três profissionais com diferentes visões sobre o tema da sustentabilidade na TI, além de um moderador que mediou as discussões entre painelistas e participantes. O workshop contou com a participação de 20 pessoas. Todos os trabalhos foram publicados online no portal de conteúdos da $\mathrm{SBC}^{11}$.

\section{PLANO DE ATIVIDADES E DURAÇÃO}

A agenda do WAIHCWS'19 terá duração de dois períodos. O primeiro período contará com uma sessão de abertura, e será reservado para as sessões técnicas dos trabalhos aceitos. O segundo será composto por uma sessão técnica e um painel no tema do workshop que envolverá os pesquisadores da comunidade de IHC que participaram da organização deste workshop ao longo desses 10 anos. Por fim, será realizada uma sessão breve de encerramento, coleta de feedback dos participantes e premiação do melhor artigo desta edição.

\section{PÚBLICO-ALVO}

O workshop se destina a pesquisadores, estudiosos e profissionais da academia e da indústria que trabalhem e tenham interesse na área de Web Social e Software Social. É de interesse do WAIHCWS receber trabalhos e reunir profissionais de áreas diversas, como Informática, Design, Educação, Direito, Ciências Sociais e Comunicação.

\section{SELEÇÃO/PUBLICAÇÃO DOS ARTIGOS}

O sistema JEMS foi utilizado para o gerenciamento das submissões e revisões dos artigos. Cada artigo foi avaliado por no mínimo três revisores e o comitê de programa foi composto por professores e pesquisadores doutores com amplo conhecimento nos tópicos de interesse do evento, muitos dos quais já participam deste comitê há alguns anos. Os anais do evento serão disponibilizados online no

\footnotetext{
10 http://ceur-ws.org/Vol-2039/

${ }^{11}$ http://portaldeconteudo.sbc.org.br/index.php/waihcws
}

Portal de Conteúdos da SBC. Os melhores artigos aceitos sejam premiados no final do workshop.

\section{DATAS SUGERIDAS}

Lançamento do site/divulgação da chamada: 28/05/2019

Prazo de submissão de artigo completo: 13/07/2019

Notificação de resultados: 17/08/2019

Envio da versão final/inscrição de autor: 31/08/2019

\section{BIOGRAFIA DOS PROPONENTES}

Kamila Rodrigues $^{12}$ é professora substituta da Universidade Federal de São Carlos (UFSCar) e na Universidade de São Paulo (USP), professora assistente da Universidade de Araraquara (UNIARA) e Universidade Paulista (UNIP), é docente do Programa de Pós-Graduação Lato Sensu no Departamento de Computação da UFSCar. Doutora em Ciência da Computação pela UFSCar (2014). Realizou Pós-doutorado no ICMC/USP. Tem atuado em pesquisas no Laboratório de Interação Flexível e Sustentável (LIFeS) no DC/UFSCar e no Laboratório Intermídia no ICMC/USP, com trabalhos que estudam a criação de documentos interativos para coleta especializada de experiências do cotidiano de indivíduos, bem como pesquisas sobre a concepção e a avaliação de interfaces de usuários com enfoque no comportamento sustentável. Tem interesse no estudo das respostas emocionais de usuários e nas suas experiências durante a interação com sistemas multimídia interativos. Orienta trabalhos voltados ao desenvolvimento de aplicações digitais terapêuticas para diferentes patologias.

Rodrigo Santos ${ }^{13}$ é professor adjunto da Universidade Federal do Estado do Rio de Janeiro (UNIRIO) e membro do Programa de PósGraduação em Informática (PPGI/UNIRIO). Doutor em Engenharia de Sistemas e Computação pela COPPE/UFRJ, tendo atuado como pesquisador visitante na University College London (2014-2015). Realizou Pós-doutorado na COPPE/UFRJ em 2016. É editor-chefe da iSys - Revista Brasileira de Sistemas de Informação, membro do Comitê-Gestor da Comissão Especial de Sistemas de Informação (CESI) da SBC, membro da SBC desde 2006 e avaliador de cursos superiores em Computação pelo INEP/MEC desde 2011. Tem experiência na área de Ciência da Computação, com ênfase em Engenharia de Software. Seus principais campos de atuação são Ecossistemas de Software, Engenharia de Requisitos e Aquisição de Software e Serviços de TI.

\section{REFERÊNCIAS}

[1] Neto, V. V., Graciano, Araujo, R, e Santos, R. P. 2017. New Challenges in the Social Web: Towards Systems-of-Information Systems Ecosystems. In Anais do WAIHCWS 2017, Joinvile, Brasil. Disponível em: http://ceur-ws.org/Vol2039/paper01.pdf. Acesso em: abril de 2019.

[2] Bannon, J. B., e Bodker, S. 2019. Reimagining participatory design. Interactions 26(1): 26-32.

[3] Bannon, J. B., Bardzell, J., e Bodker, S. 2018. Introduction: Reimagining Participatory Design - Emerging Voices. ACM Trans. Comput. - Hum. Interact. 25(1): $1: 1-1: 8$.

\footnotetext{
${ }^{12}$ http://lattes.cnpq.br/9035684997439778

13 http://lattes.cnpq.br/8613736894676086
} 